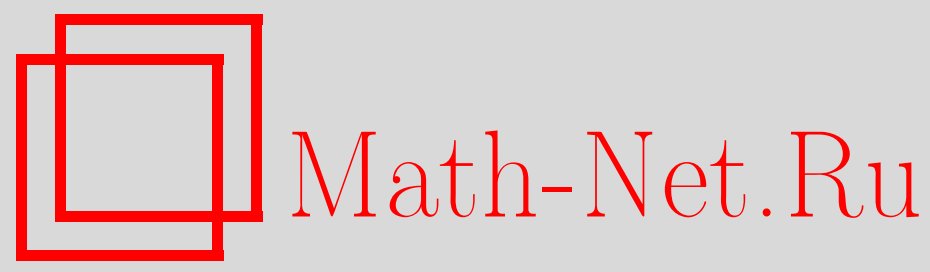

А. А. Амиршадян, Граничная интерполяционная задача в классах обобщенных неванлинновских матриц-функций, Матем. заметки, 2003, том 73, выпуск 2, 173-178

DOI: https://doi.org/10.4213/mzm175

Использование Общероссийского математического портала Math-Net.Ru подразумевает, что вы прочитали и согласны с пользовательским соглашением http://www.mathnet.ru/rus/agreement

Параметры загрузки:

IP : 54.198 .187 .58

26 апреля 2023 г., 03:36:52

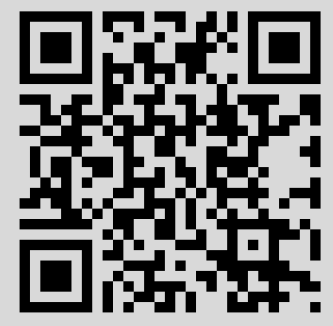




\section{ГРАНИЧНАЯ ИНТЕРПОЛЯЦИОННАЯ ЗАДАЧА В КЛАССАХ ОБОБЩЕННЫХ НЕВАНЛИННОВСКИХ МАТРИЦ-ФУНКЦИЙ}

\section{А. А. Амиршадян}

В работе изучается граничная индефинитная интерполяционная проблема в классах обобщенных неванлинновских матриц-функций. Установлено биективное соответствие между множеством всех решений рассматриваемой задачи и классом так называемых $G$-регулярных самосопряженных расширений модельного симметрического оператора, связанного с задачей. Получены достаточные условия $G$-регулярности самосопряженных расширений (в терминах функции Вейля) и формула для описания всех решений рассматриваемой задачи.

Библиография: 15 названий.

Скалярная граничная проблема Неванлинны-Пика в классе сжимающих функций рассматривалась в [1], а для классов Неванлинны и Стилтьеса - в [2], [3]. Матричная граничная проблема с кратными точками интерполящии рассматривалась в [4]. В настоящей работе в рамках операторного подхода изучается граничная индефинитная интерполящионная проблема в классах $N_{\kappa}\left(\mathbb{C}^{n}\right)$ обобщенных неванлинновских матриц-функций. Установлено биективное соответствие между множеством всех решений рассматриваемой задачи и классом так называемых $G$-регулярных самосопряженных расширений модельного симметрического оператора, связанного с задачей. Получены достаточные условия $G$-регулярности самосопряженных расширений в терминах функции Вейля. Другой подход к этой задаче, связанньй с индефинитньм вариантом теоремы Берлинга-Лакса, применялся в [5].

ОПРЕДЕЛЕниЕ 1. Пара $(n \times n)$-матриц-функций $\{\phi(\lambda), \psi(\lambda)\}$, голоморфных в области $\mathscr{O}=\overline{\mathscr{O}} \subset \mathbb{C} \backslash \mathbb{R}$, назьвается обобщенной неванлинновской парой (или $N_{\kappa}$-парой, $\left.\kappa \in \mathbb{Z}_{+}\right)$, если

(1) ядро $\mathbf{N}_{\phi \psi}(\lambda, \mu)=\left(\phi(\mu)^{*} \psi(\lambda)-\psi(\mu)^{*} \phi(\lambda)\right) /(\lambda-\bar{\mu})$ имеет $\kappa$ отрицательных квадратов в $\mathscr{O}$;

(2) $\psi(\bar{\lambda})^{*} \phi(\lambda)-\phi(\bar{\lambda})^{*} \psi(\lambda)=0$ для всех $\lambda \in \mathscr{O}$;

(3) $\operatorname{rank}\left\{\phi(\lambda)^{*}: \psi(\lambda)^{*}\right\}=n$ для всех $\lambda \in \mathscr{O}$.

Две пары $\{\phi, \psi\}$ и $\left\{\phi_{1}, \psi_{1}\right\}$ назьваются әквивалентными, если $\phi_{1}(\lambda)=\phi(\lambda) H(\lambda)$, $\psi_{1}(\lambda)=\psi(\lambda) H(\lambda)$ для некоторой голоморфной и обратимой в $\mathscr{O}$ матришы-функции $H(\lambda)$. Множество эквивалентных $N_{\kappa}$-пар обозначим $\widetilde{N}_{\kappa}\left(\mathbb{C}^{n}\right)$. Класс $N_{\kappa}\left(\mathbb{C}^{n}\right)$ матриц-функций рассматриваем как подмножество $\tilde{N}_{\kappa}\left(\mathbb{C}^{n}\right)$, отождествляя матрицу-функцию $F(\lambda)$ с парой $\{I, F(\lambda)\}$. Напомним, что пространством Понтрягина назьвается гильбертово 
пространство П, снабженное индефинитным скалярньп произведением $[\cdot, \cdot]=(J \cdot, \cdot)$, где $J=J^{*}=J^{-1}$ - линейный оператор в П с конечным отрицательным индексом sq_ $\Pi=\operatorname{dim}(I-J) \Pi$. Пусть $S$ - замкнутый не плотно заданньй симметрический линейный оператор в пространстве Понтрягина $(\Pi,[\cdot, \cdot])$ с непустым множеством $\hat{\rho}(S)$ точек регулярного типа такой, что дефектные подпространства $\mathcal{N}_{\lambda}=\operatorname{ker}\left(S^{+}-\lambda\right), \lambda \in \hat{\rho}(S)$, конечномерны и индексы дефекта равны: $n_{+}(S)=n_{-}(S)\left(=\operatorname{dim} \mathcal{N}_{\lambda}\right), \lambda \in \mathbb{C}_{ \pm} \cap \hat{\rho}(S)$. Здесь $S^{+}$- линейное отношение в $\Pi$, сопряженное к оператору $S$.

ОПРЕДЕЛЕНИЕ 2 [6]. Совокупность $\left\{\mathscr{H}, \Gamma_{0}, \Gamma_{1}\right\}$, где $\mathscr{H}$ - вспомогательное гильбертово пространство, а $\Gamma_{0}, \Gamma_{1}$ - линейные отображения из $S^{+}$в $\mathscr{H}$, называется граничной тройкой отношения $S^{+}$, если отображение $\Gamma: \hat{f} \rightarrow\left\{\Gamma_{0} \hat{f}, \Gamma_{1} \hat{f}\right\}$ из $S^{+}$в $\mathscr{H} \oplus \mathscr{H}$ является сюръективным и для всех $\hat{f}=\left\{f, f^{\prime}\right\}, \hat{g}=\left\{g, g^{\prime}\right\} \in S^{+}$выполняется соотношение

$$
\left[f^{\prime}, g\right]-\left[f, g^{\prime}\right]=\left(\Gamma_{1} \hat{f}, \Gamma_{0} \hat{g}\right) \mathscr{H}-\left(\Gamma_{0} \hat{f}, \Gamma_{1} \hat{g}\right) \mathscr{H}
$$

С каждой граничной тройкой естественно связаны два самосопряженных расширения $A_{j}=\operatorname{ker} \Gamma_{j}, j=0,1$ и голоморфные в $\rho\left(A_{0}\right)$ оператор-функции $M(\lambda)$ и $\gamma(\lambda)$ со значениями в $[\mathscr{H}]$ и $\left[\mathscr{H}, \mathcal{N}_{\lambda}\right]$, назьваемые функиией Вейля и $\gamma$-полем оператора $S$ соответственно. Последние определяются равенствами

$$
\hat{\gamma}(\lambda)=\left(\Gamma_{0} \mid \widehat{\mathcal{N}}_{\lambda}\right)^{-1}, \quad M(\lambda)=\Gamma_{1} \hat{\gamma}(\lambda), \quad \gamma(\lambda)=\pi_{1} \hat{\gamma}(\lambda)
$$

где $\widehat{\mathcal{N}}_{\lambda}=\left\{\left\{f_{\lambda}, \lambda f_{\lambda}\right\}: f_{\lambda} \in \mathscr{N}_{\lambda}\right\}$, а $\pi_{1}-$ проектор на первую компоненту $\widehat{\mathscr{N}}_{\lambda}$ (см. [7]-[9]). В [7], [8] показано, что функция Вейля $M(\lambda)$ является $Q$-функцией оператора $S$ в смысле [10], т.е. справедливо тождество

$$
M(\lambda)-M(\mu)=(\lambda-\mu) \gamma(\bar{\lambda})^{+} \gamma(\mu), \quad \lambda, \mu \in \rho\left(A_{0}\right)
$$

Пусть $\widetilde{A}$ - самосопряженное расширение оператора $S$ в пространстве Понтрягина $\widetilde{\Pi}=$ $\Pi \oplus \Pi^{\prime}$. Обобщенную резольвенту

$$
\mathbf{R}_{\lambda}=\left.P_{\Pi}(\widetilde{A}-\lambda)^{-1}\right|_{\Pi}, \quad \lambda \in \rho(\widetilde{A}),
$$

относят к классу $\Omega_{\kappa^{\prime}}(S)$, если sq_ $\Pi^{\prime}=\kappa^{\prime}$ и вьполнено условие минимальности:

$$
\overline{\operatorname{span}}\left\{\Pi+(\widetilde{A}-\lambda)^{-1} \Pi \mid \lambda \in \rho(\widetilde{A})\right\}=\widetilde{\Pi}
$$

Если вьполнено условие минимальности $(3)$ и $\mathrm{sq}_{-} \Pi^{\prime}=0$, то $\widetilde{A}$ называют регулярным расиирением. Если $\lambda_{0} \in \rho\left(A_{0}\right)$ и $M^{\prime}\left(\lambda_{0}\right)>\underset{\widetilde{A}}{0}$, то для любого регулярного самосопряженного расширения $\widetilde{A}$ подпространства $\operatorname{ker}\left(\widetilde{A}-\lambda_{0}\right)$ являются равномерноположительными [10].

Следующая теорема в случае $\overline{\operatorname{dom}} S=\Pi$ и sq_ $\Pi^{\prime}=0$ получена в [10], а в случае $\overline{\mathrm{dom}} S \neq \Pi-$ в [9]. 
ТЕОРема 3. Пусть $S$ - замкнутый симметрический оператор в пространстве Понтрягина $\Pi,\left\{\mathscr{H}, \Gamma_{0}, \Gamma_{1}\right\}$ - граничная тройка линейного отношения $S^{+}, M(\lambda)$ соответствующая функция Вейля. Тогда равенство

$$
\mathbf{R}_{\lambda}=\left(A_{0}-\lambda\right)^{-1}-\gamma(\lambda) \phi(\lambda)(\psi(\lambda)+M(\lambda) \phi(\lambda))^{-1} \gamma(\bar{\lambda})^{+}
$$

устанавливает биективное соответствие мехду мнохеством обобщенных резольвент класса $\Omega_{\kappa^{\prime}}(S)$, голоморфных в точке $\lambda_{0} \in \rho\left(A_{0}\right)$, и множеством $N_{\kappa^{\prime}}$ nap $\{\phi(\lambda), \psi(\lambda)\}$ maкux, чmо $\operatorname{det}\left(\psi\left(\lambda_{0}\right)+M\left(\lambda_{0}\right) \phi\left(\lambda_{0}\right)\right) \neq 0$.

Лемма 4. Пусть $\left\{\mathscr{H}, \Gamma_{0}, \Gamma_{1}\right\}$ - граничная тройка отношения $S^{+}$такая, что $A_{0}$ - оператор. Если $\widetilde{A}=\widetilde{A}^{+}-$минимальное самосопряженное расширение оператора $S$, действующее в пространстве Понтрягина $\widetilde{\Pi} \supseteq \Pi, \tilde{\kappa}:=$ sq $_{-} \widetilde{\Pi} \geqslant \kappa:=$ sq $_{-} \Pi$, $u\{\phi(\lambda), \psi(\lambda)\}-N_{\tilde{\kappa}-\kappa}-$ пара, соответствующ, ая расиирению $\widetilde{A}$ в силу (4), то равномерная положительность (отрицательность) подпространства $\operatorname{ker}\left(\widetilde{A}-\lambda_{0}\right)$, $\lambda_{0} \in \rho\left(A_{0}\right)$, әквивалентна условию

$$
\underset{\lambda \rightarrow \lambda_{0}}{\lim }\left(\lambda-\lambda_{0}\right) \phi(\lambda)(M(\lambda) \phi(\lambda)+\psi(\lambda))^{-1} \geqslant 0 \quad(\leqslant 0)
$$

В случае $\lambda_{0}=\infty$ условие (5) принимает вид

$$
\lim _{\lambda \rightarrow \infty} \frac{\phi(\lambda)(M(\lambda) \phi(\lambda)+\psi(\lambda))^{-1}}{\lambda} \geqslant 0 \quad(\leqslant 0) .
$$

Условие $\lambda_{0} \notin \sigma_{p}(\widetilde{A})$ эквивалентно условию $(5)$, в котором имеет место точное равенство. В том случае, когда $\Pi, \widetilde{\Pi}-$ гильбертовы пространства и $\lambda_{0}=\infty$, аналогичньй вариант этого равенства имеется в [7] (см. также [11]).

Рассматривается следующая

ЗАДАчА $\partial \mathrm{IP}_{\kappa}$. Даны вещественные точки $z_{j}$ и симметрические матрицы $W_{j}, D_{j}$, $j=1, \ldots, m$. Требуется найти матрицу-функцию $F(\lambda) \in N_{\kappa}\left(\mathbb{C}^{n}\right)$, удовлетворяющую условиям

$$
\begin{aligned}
& \lim _{\lambda \rightarrow z_{j}} F(\lambda)=W_{j}, \quad j=1, \ldots, m, \\
& \frac{\lim }{\lambda \rightarrow z_{j}} F^{\prime}(\lambda) \leqslant D_{j}, \quad j=1, \ldots, p
\end{aligned}
$$

Предполагается, что некасательные пределы в $(7),(8)$ существуют. Условие разрешимости задачи формулируется в терминах матришы Пика $\mathbf{P}=\left(P_{j k}\right)_{j, k=1}^{m}$, имеюшей вид [1], [2]

$$
P_{j k}= \begin{cases}\frac{W_{j}-W_{k}}{z_{j}-z_{k}} & \text { при } j \neq k, \\ D_{j}, & \text { при } j=k .\end{cases}
$$


В дальнейшем считаем, что $\operatorname{det} \mathbf{P} \neq 0$. При получении основной теоремы используется функциональная модель Крейна-Лангера [12] и Надя-Кораньи [13] (см. также [2]). А именно, рассматривается пространство П формальных сумм

$$
\Pi=\left\{f(t) \mid f(t)=\sum_{j=1}^{m} \varepsilon_{j} f_{j}=\sum_{j=1}^{m} \frac{t-z_{1}}{t-z_{j}} f_{j}, f_{j} \in \mathbb{C}^{n}, t \in \mathbb{C} \backslash\left\{z_{1}, \ldots, z_{m}\right\}\right\}
$$

со скалярным произведением

$$
[f(t), g(t)]=\sum_{j, k}^{m} g_{k}^{*} P_{j k} f_{j}
$$

Пространство П является пространством Понтрягина ( $\left.\mathrm{sq}_{-} \Pi=\mathrm{sq}_{-} \mathbf{P}\right)$. На подпространстве $\overline{\operatorname{dom}} S=\left\{f(t) \mid \sum_{j=1}^{m} f_{j}=0\right\}$ определяется модельный оператор $S$ умножения на независимую переменную:

$$
S f(t)=t f(t)=\sum_{j=1}^{m} \varepsilon_{j} z_{j} f_{j}
$$

Оператор $S$ является симметрическим с индексами дефекта $n_{+}(S)=n_{-}(S)=n$. Пусть $G$ - оператор вложения $\mathbb{C}^{n}$ в $\Pi$, т.е. $G f:=\varepsilon_{1} f, f \in \mathbb{C}^{n}$. Для произвольного минимального самосопряженного расширения $\widetilde{A}$ оператора $S$, действуюшего в пространстве Понтрягина $\widetilde{\Pi} \supseteq \Pi, \mathrm{sq}_{-} \widetilde{\Pi} \geqslant \mathrm{sq}_{-} \Pi$, обозначим

$$
\Pi_{G}:=\overline{\operatorname{span}}\left\{\left(I+\left(\lambda-z_{1}\right)(\widetilde{A}-\lambda)^{-1}\right) G f \mid \lambda \in \rho(\widetilde{A}), f \in \mathbb{C}^{n}\right\}
$$

ОпРЕДЕЛЕниЕ 5. Минимальное самосопряженное расширение $\widetilde{A}$ будем назьвать G-регулярным, если $\mathrm{sq}_{-} \widetilde{\Pi}=\mathrm{sq}_{-} \Pi_{G}($ см. (9)).

Следующая теорема является основной. Она устанавливает биективное соответствие между всеми решениями задачи $\partial \mathrm{IP}_{\kappa}$ и соответствуюшими $G$-регулярными расширениями модельного оператора $S$.

Теорема 6. Пусть матрица $\mathbf{P}$ невырожсдена $u \mathrm{sq}_{-} \mathbf{P} \leqslant \kappa$. Для того чтобь функиия $F(\lambda)$ являлась решением задачи $\mathrm{IP}_{\kappa}$, необходимо и достаточно, чтобь она имела вид

$$
F(\lambda)=W_{1}+\left(\lambda-z_{1}\right) G^{+}\left(I+\left(\lambda-z_{1}\right)(\widetilde{A}-\lambda)^{-1}\right) G
$$

где $\widetilde{A}\left(=\widetilde{A}^{+}\right)$- произвольное $G$-регулярное расширение оператора $S$, действующее в пространстве Понтрягина $\widetilde{\Pi} \supseteq \Pi$, sq- $\widetilde{\Pi}=\kappa$, такое, что подпространства $\operatorname{ker}\left(\widetilde{A}-z_{j}\right)$ являются равномерно положительными при $j=1, \ldots, m$.

ЛЕмма 7. Если $z_{j} \in \rho(\widetilde{A}), j=1, \ldots, m$, то минимальность самосопряжсенного расширения $\widetilde{A}$ әквивалентна его $G$-регулярности. 
СлЕДСтВИЕ 8. Пусть $\operatorname{det} \mathbf{P} \neq 0$. Тогда условие sq_ $_{-} \mathbf{P} \leqslant \kappa$ является необходимым и достаточным для разрешимости задачи $\partial \mathrm{IP}_{\kappa}$.

Утверждение следствия 8 следует из леммы 7 и [14, предложение 1.9]. Таким образом, в случае $z_{j} \in \rho(\widetilde{A}), j=1, \ldots, m$, описание всех решений задачи $\partial \mathrm{IP}_{\kappa}$ сводится к описанию соответствуюших минимальных самосопряженных расширений оператора $S$ (см. (10)) и может быть легко получено из теоремы 3 . Заметим, что тогда в условиях (8) будут иметь место точные равенства. Эффективное описание всех $G$-регулярных расширений нам не известно, однако в лемме 9 и следствии 10 приводятся простые достаточные условия $G$-регулярности расширения $\widetilde{A}$ модельного оператора $S$.

Лемма 9. Пусть sq_ $\mathbf{P}=\kappa, \widetilde{A}-$ минимальное регулярное самосопряжсенное расширение оператора $S$, действующее в пространстве Понтрягина $\widetilde{\Pi} \supseteq \Pi$, такое, что подпространства $\operatorname{ker}\left(\widetilde{A}-z_{j}\right)$ равномерно полохсительны, $j=1, \ldots, m$. Тогда расширение $\widetilde{A}$ является $G$-регулярным.

СледСтвие 10. Пусть sq_ $\mathbf{P}=\kappa, z_{j} \in \rho\left(A_{0}\right) u M^{\prime}\left(z_{j}\right)>0, j=1, \ldots, m$. Тогда любое минимальное регулярное самосопря⿻енное расширение $\widetilde{A}$ оператора $S$ является $G$-регулярным.

При описании решений задачи $\partial \mathrm{IP}_{\kappa}$ используются методы теории представлений Крейна [15] и формула резольвентной матрицы, полученная в [8], [9]. Выбирая определенньм образом граничную тройку [14], находим вид функции Вейля $M(\lambda)$ и ее производной:

$$
M(\lambda)=V\left((Z-\lambda) \mathbf{P}+V^{*} W\right)^{-1} V^{*}:=V \Phi_{\lambda}^{-1} V^{*}, \quad M^{\prime}(\lambda)=V \Phi_{\lambda}^{-1} \mathbf{P} \Phi_{\lambda}^{-1} V^{*} .
$$

Здесь $V=\left(I_{n}, \ldots, I_{n}\right), W=\left(W_{1}, \ldots, W_{m}\right), Z=\operatorname{diag}\left(z_{1} I_{n}, \ldots, z_{m} I_{n}\right)$.

Условие $\operatorname{det} \Phi_{\lambda} \neq 0$ эквивалентно условию $\lambda \in \rho\left(A_{0}\right)$. Теперь определим матрицу решений $\Omega(\lambda)$ задачи $\partial \mathrm{IP}_{\kappa}$ :

$$
\Omega(\lambda)=\left(\Omega_{i j}\right)_{i, j=1}^{2}=I_{2 n}+\left(\begin{array}{c}
W \\
V
\end{array}\right)(Z-\lambda)^{-1} \mathbf{P}^{-1}\left(-V^{*}, W^{*}\right) .
$$

Вид матрицы решений $(12)$ для задачи $\partial \mathrm{IP}_{\kappa}$ совпадает с хорошо известньм видом матрицы решений классической интерполящионной проблемы Неванлинны-Пика ([4], см. также [14]). Если в условиях (8) $p=m$, то, используя леммы 4,9 , получаем следующую теорему.

Tеорема 11. Пусть sq $-\mathbf{P}=\kappa, \operatorname{det} \Phi_{z_{j}} \neq 0, j=1, \ldots, m, p=m$. Тогда формула

$$
F(\lambda)=\left(\Omega_{12}(\lambda) \psi(\lambda)-\Omega_{11}(\lambda) \phi(\lambda)\right)\left(\Omega_{22}(\lambda) \psi(\lambda)-\Omega_{21}(\lambda) \phi(\lambda)\right)^{-1}
$$

устанавливает взаимно однозначное соответствие мехуу множеством решений задачи $\partial \mathrm{IP}_{\kappa}$ и множеством $N_{0}$-nар $\{\phi(\lambda), \psi(\lambda)\}$ таких, что

$$
\begin{gathered}
\operatorname{det}\left(\Omega_{22}(\lambda) \psi(\lambda)-\Omega_{21}(\lambda) \phi(\lambda)\right) \not \equiv 0 \\
\lim _{\lambda \rightarrow z_{j}}\left(\lambda-z_{j}\right) \phi(\lambda)\left(V \Phi_{\lambda}^{-1} V^{*} \phi(\lambda)+\psi(\lambda)\right)^{-1} \geqslant 0, \quad j=1, \ldots, m .
\end{gathered}
$$

ЗАмечАние 12 . В том случае, когда $M^{\prime}\left(z_{j}\right)=V \Phi_{z_{j}}^{-1} \mathbf{P} \Phi_{z_{j}}^{-1} V^{*}>0, j=1, \ldots, m$ (см. (11)), условия (15) выполняются автоматически (см. следствие 10). 


\section{СПИСОК ЦИТИРОВАННОЙ ЛИТЕРАТУРЫ}

[1] Sarason D. Nevanlinna-Pick interpolation with boundary data // Integral Equations Operator Theory. 1998. V. 30. P. 231-250.

[2] Alpay D., Dijksma A., Langer H. Classical Nevanlinna-Pick interpolation with real interpolation points // IWI-preprint 1998-3-02: Groningen University, 1998.

[3] Кацнельсон В.Э. Интерполяция "на спектре" в классе функций Стилтьеса (случай одного узла) // Функцион. анализ и прикладная матем. 1982. С. 33-42.

[4] Нудельман А.А. Многоточечная матричная проблема моментов // Докл. АН СССР. 1988. T. 298. № 4. C. 812-815.

[5] Ball J. A., Helton J. W. Interpolation problem of Pick-Nevanlinna and Loewner types for meromorphic matrix functions: parametrization of the set of all solutions // Integral Equations Operator Theory. 1986. V. 9. P. 155-203.

[6] Горбачук М. Л., Горбачук В. И. Граничные задачи для дифференциально-операторных уравнений. Киев: Наукова думка, 1984.

[7] Маламуд М. М. О формуле обобщенных резольвент неплотно заданного эрмитова оператора // Укр. матем. ж. 1992. Т. 44. № 12. С. 1658-1688.

[8] Derkach V., Malamud M. The extension theory of hermitian operators and the moment problem // J. Math. Sci. 1995. V. 73. №2. P. 141-242.

[9] Derkach V. On generalized resolvents of hermitan relations in Krein spaces // J. Math. Sci. 1999. V. 97. № 5. P. 4420-4460.

[10] Крейн М. Г., Лангер Г. К. О дефектных подпространствах и обобщенных резольвентах эрмитова оператора в пространстве $\Pi_{\kappa} / /$ Функцион. анализ и его прилож. 1971. Т. 5. № 2 . C. 59-71; № 3. C. 54-69.

[11] Derkach V., Hassi S., Malamud M., Snoo H.S. V. Generalized resolvents of symmetric operators and admissibility // Preprint № 252. Helsinki: University of Helsinki, 2000.

[12] Krein M. G., Langer H. Über die $Q$-Function eines $\pi$-hermiteschen Operator in Raume $\Pi_{\kappa}$ // Acta Sci. Math. 1973. V. 34. P. 191-230.

[13] Nagy Sz., Koranyi A. Relations d'une problème de Nevanlinna et Pick avec la théorie des opérateurs de l'espace hilbertien // Acta Math. Acad. Hung. 1956. V. 7. P. 295-303.

[14] Amirshadyan A., Derkach V. Interpolation in generalized Nevanlinna and Stieltjes classes // J. Operator Theory. 1999. V. 42. P. 145-188.

[15] Krein M. G. Основные положения теории представления эрмитовых операторов с индексом дефекта $(m, m) / /$ Укр. матем. ж. 1949. Т. 1. № 2. С. 3-66. 